Accelerator Division

Alternating Gradient Synchrotron Department

BROOKHAVEN NATIONAL LABORATORY

Upton, New York 11973

Accelerator Division

Technical Note

AGS/AD/Tech. Note No. 367

\title{
BTA Stripping Foil Studies
}

\section{T.Roser}

October 2, 1992

Stripping efficiencies were measured for $277 \mathrm{MeV} / \mathrm{amu} \mathrm{Au}^{33+}$ ions with Copper foils ranging in thickness from $25 \mu \mathrm{m}$ to $100 \mu \mathrm{m}$. The beam was analyzed with part of the BTA beam line consisting of QH4, D2, QV5, D3, and QH6, and the multi-wire profile monitor MW060. The relative charge state abundances were studied to find the optimum foil thickness. It was also possible to extract electron stripping and pick-up cross sections. 
The $\mathrm{Au}^{33+}$ beam was extracted from the AGS Booster at a momentum of $770 \mathrm{MeV} / \mathrm{c} / \mathrm{amu}(277 \mathrm{MeV} / \mathrm{amu}$ kinetic energy). Typically a $50 \mu \mathrm{m}$ thick Copper foil was used to strip $\mathrm{Au}^{33+}$ to $\mathrm{Au}^{78+}$, which was then injected into the AGS for acceleration to $10 \mathrm{GeV} / \mathrm{amu}$. By varying the dipole magnets D2 and D3 following the stripping foil the relative abundance of the charge states $\mathrm{Au}^{77+}, \mathrm{Au}^{78+}$ and $\mathrm{Au}^{79+}$ could be studied with the multi-wire profile monitor MW060 . Measurements were made for $25,37.5,50$, and $100 \mu \mathrm{m}$ thick foils each consisting of about three different settings of the analyzing magnet. Fig. 1 shows a typical profile taken with the $37.5 \mu \mathrm{m}$ thick foil. For some of the profiles all the odd-numbered wires showed an increased reading. We found that this was due to the fact that part of the beam was hitting the read-out wires, that were strung on either side of the active area towards the vacuum feed through located at the top. For the following analysis, we therefore used only the readings from the even-numbered wires which always gave reliable results for all measurements.

One profile alone did not cover the full charge state distribution. Therefore, several profiles had to be taken with different settings for the analyzing magnet as mentioned above. The profiles for different magnet settings were then combined by fitting all profiles with a single distribution made up from three gaussians corresponding to the three charge states $77+, 78+$, and $79+$. The gaussian distributions all had the same width and were separated by equal distances. The results are shown in Figures 2 - 5 .

The overlap between the profiles taken with different magnets settings was sufficiently large that the magnet calibration could be included as fitting parameter. The average over all foil measurements was $0.915 \mathrm{~mm} / A$. The separation between the gaussian distributions was $36.5 \pm 0.3 \mathrm{~mm}$ in good agreement with the expected dispersion of $38.5 \mathrm{~mm}$ at MW060 calculated from the settings of the beam elements. The measured value can be used to calibrate the position at the profile monitor in terms of beam momentum. The result is $0.27 \frac{\mathrm{MeV} / \mathrm{c} / \mathrm{amu}}{\mathrm{mm}}$. The following table gives the remaining fitting parameters for the four foil measurements:

\begin{tabular}{|l|c|c|c|c|}
\hline Foil Thickness $[\mu \mathrm{m}]$ & 25 & 37.5 & 50 & 100 \\
\hline Center of $77+$ Peak $[\mathrm{mm}]$ & $22.6 \pm 0.2$ & $7.2 \pm 0.2$ & $3.9 \pm 0.2$ & $-18.7 \pm 0.3$ \\
Width of Peaks [mm] & $8.2 \pm 0.3$ & $9.1 \pm 0.2$ & $10.7 \pm 0.3$ & $13.6 \pm 0.4$ \\
Abundance of $77+[\%]$ & $51 \pm 5$ & $13 \pm 1$ & $9 \pm 1$ & $4 \pm 9$ \\
Abundance of $78+[\%]$ & $40 \pm 5$ & $44 \pm 1$ & $46 \pm 1$ & $42 \pm 4$ \\
Abundance of $79+[\%]$ & $10 \pm 1$ & $44 \pm 1$ & $45 \pm 1$ & $54 \pm 5$ \\
\hline
\end{tabular}

From the variation of the peak position of charge state $77+$ with the foil 
thickness one can extract the energy loss in the Copper foil. As shown in Fig. 6 the average slope is $0.52 \mathrm{~mm} / \mu \mathrm{m}$ which corresponds to an energy loss of $880 \frac{\mathrm{MeV} / \mathrm{amu}}{\mathrm{cm}}$ or $99 \frac{\mathrm{MeV} / \mathrm{amu}}{\mathrm{g} / \mathrm{cm}^{2}}$. The expected value obtained from the Bethe-Bloch equation[1], which leads to the scaling law

$$
\left.\frac{d E}{d x}\right|_{A X^{Z}}=\frac{Z^{2}}{A} \times\left.\frac{d E}{d x}\right|_{\text {proton }}\left(p=\frac{p_{X}}{A}\right)
$$

is $80 \frac{\mathrm{MeV} / \mathrm{amu}}{\mathrm{g} / \mathrm{cm}^{2}}$, in good agreement with the measured value. The total energy loss for the $100 \mu \mathrm{m}$ foil was therefore about $9 \mathrm{MeV} / \mathrm{amu}$.

The increase of the width of individual charge state peaks is due to multiple scattering in the foil which leads to an increase of the divergence and of the energy spread of the beam. In gaussian approximation the multiple scattering adds in quadrature to the initial beam width which results in the following dependency of the width $\sigma$ on the foil thickness $d$ :

$$
\sigma=\sqrt{\sigma_{0}^{2}+\alpha d}
$$

Fig. 7 shows the data together with the fitted thickness dependency according the eq. (2). The result of the fit is $\sigma_{0}=4.8 \mathrm{~mm}$ and $\alpha=1.7 \frac{\mathrm{mm}^{2}}{\mu \mathrm{m}}$. The contribution to $\alpha$ from increased divergence based on the gaussian approximation to Moliere's theory[2] is only $0.92 \frac{\mathrm{mm}^{2}}{\mu m}$, where we used the calculated projection factor of $9.8 \frac{\mathrm{mm}}{\mathrm{mrad}}$ for the beam transport between foil and monitor. This suggests that half of the increase in the beam width is due to increased energy spread.

Clearly the foil leads to a significant beam emittance blow-up of up to a factor of 8. A more optimized situation should include a thinner foil, as discussed below, and a smaller beam spot at the foil.

The relative abundance of the three charge states can be understood within the framework of a simple model if we assume that after a short distance $d_{0}$ all electrons except the $\mathrm{K}$ shell electrons are stripped off. Beyond this initial stripping foil thickness the relative abundances of the three charge states are then determined solely by single electron pick-up and stripping between the three charge states $77+, 78+$, and $79+[3]$. This can be described by a set of coupled differential equations:

$$
\frac{d}{d x}\left(\begin{array}{c}
r_{79} \\
r_{78} \\
r_{77}
\end{array}\right)=\left(\begin{array}{ccc}
-p_{1} & s_{1} & 0 \\
p_{1} & -\left(s_{1}+p_{1}\right) & s_{2} \\
0 & p_{2} & -s_{2}
\end{array}\right)\left(\begin{array}{c}
r_{79} \\
r_{78} \\
r_{77}
\end{array}\right)
$$


where $r_{79}, r_{78}$, and $r_{77}$ are the relative abundances as listed in Table $1 . s_{1}$ and $s_{2}$ are stripping probabilities for $78 \rightarrow 79$ and $77 \rightarrow 78$, respectively, whereas $p_{1}$ and $p_{2}$ are the pick-up probabilities for $79 \rightarrow 78$ and $78 \rightarrow 77$, respectively. The system of linear differential equations can easily be solved. The eigenvalues are:

$$
\begin{gathered}
\lambda_{1}=0 \\
\lambda_{2}=\frac{a-\left(p_{1}+p_{2}+s_{1}+s_{2}\right)}{2} \\
\lambda_{3}=\frac{-a-\left(p_{1}+p_{2}+s_{1}+s_{2}\right)}{2}
\end{gathered}
$$

with

$$
a^{2}=p_{1}^{2}+p_{2}^{2}+s_{1}^{2}+s_{2}^{2}-2 p_{1} p_{2}+2 p_{1} s_{1}+2 p_{2} s_{1}-2 p_{1} s_{2}+2 p_{2} s_{2}-2 s_{1} s_{2}
$$

A fit to the data is shown in Fig. 8 and gives the following results:

$$
\begin{aligned}
& d_{0}=22.4 \pm 0.5 \mu m^{-1} \\
& s_{1}=0.20 \pm 0.04 \mu m^{-1} \\
& s_{2}=0.27 \pm 0.07 \mu m^{-1} \\
& p_{1}=0.19 \pm 0.04 \mu m^{-1} \\
& p_{2}=0.06 \pm 0.02 \mu m^{-1}
\end{aligned}
$$

This simple model describes the dependency of the abundances on foil thickness very well, which is reflected in a confidence level of $40 \%$ of the $\chi^{2}$ distribution. For a very thick foil these probabilities result in asymptotic abundances of

$$
\begin{aligned}
& r_{77}=10 \% \\
& r_{78}=44 \% \\
& r_{79}=46 \%
\end{aligned}
$$

The probabilities calculated above can be used to determine absolute cross sections:

$$
\begin{array}{ccc} 
& \text { This experiment } & \text { Predicted } \\
\sigma(78 \rightarrow 79) & (24 \pm 5) \times 10^{-21} \mathrm{~cm}^{2} & 9 \times 10^{-21} \mathrm{~cm}^{2} \\
\sigma(77 \rightarrow 78) & (32 \pm 8) \times 10^{-21} \mathrm{~cm}^{2} & 22 \times 10^{-21} \mathrm{~cm}^{2} \\
\sigma(79 \rightarrow 78) & (22 \pm 5) \times 10^{-21} \mathrm{~cm}^{2} & 12 \times 10^{-21} \mathrm{~cm}^{2} \\
\sigma(78 \rightarrow 77) & (7 \pm 2) \times 10^{-21} \mathrm{~cm}^{2} & 6 \times 10^{-21} \mathrm{~cm}^{2}
\end{array}
$$

The predicted cross sections are based on the relativistic Bethe-Bloch theory[1] for the stripping cross sections and on an extrapolation of eikonal calculations of nonradiative-capture for a $\mathrm{Xe}$ projectile on a $\mathrm{Cu}$ target[4]. Note that the prediction for radiative capture is a factor of 10 smaller for a heavy target such as $\mathrm{Cu}$. 
This analysis shows that, even with this relative high energy beam, it is possible to produce $\mathrm{Au}^{77+}$ ions with high efficiency. This is be achieved with a thin $22 \mu \mathrm{m}$ thick foil which also introduces only minimal emittance growth.

\section{References}

[1] H.A.Bethe, Ann.Phys. (Leipzig) 5, 325 (1930); C.Moller, Ann.Phys. (Leipzig) 14, 531 (1932)

[2] W.T.Scott, Rev.Mod.Phys. 35, 231 (1963)

[3] H.Gould et al., Phys.Rev.Lett. 52, 180 (1984)

[4] W.E.Meyerhof et al., Phys.Rev. A 32, 3291 (1985) 


\section{Figure Captions}

Fig.1: Beam profile from multi wire monitor MW060 for a foil thickness of $37.5 \mu \mathrm{m}$.

Fig.2: Fit to the charge state distribution of the measurements with the $25 \mu \mathrm{m}$ thick Copper foil.

Fig.3: Fit to the charge state distribution of the measurements with the $37.5 \mu \mathrm{m}$ thick Copper foil.

Fig.4: Fit to the charge state distribution of the measurements with the $50 \mu \mathrm{m}$ thick Copper foil.

Fig.5: Fit to the charge state distribution of the measurements with the $100 \mu \mathrm{m}$ thick Copper foil.

Fig.6: Position of $\mathrm{Au}^{77+}$ peak vs. foil thickness. The straight line represents a fit to the data of a constant energy loss per foil thickness.

Fig.7: Width of charge state peaks vs. foil thickness. The line represents a fit to the data based on energy independent contributions to the beam width from multiple scattering which are then summed in quadrature.

Fig.8: Relative charge state abundance vs. foil thickness. The $\mathrm{Au}^{79+}, \mathrm{Au}^{78+}$, and $\mathrm{Au}^{77+}$ abundances are shown as circles, squares and crosses and the model calculation as solid line, dashed line, and dashed-dotted line, respectively. The model is based on rate equations between these three charge states only. 
** Current Settings Only "*

Data type: raw - baseline Cycle display: average Group display: average Groups to acquire: 1 PPM: Heavy lons 3

Gain: HIG

Integrator start: 10010 usec

Hor. wires: 32

Ver. wires: 32

Hor. spacing: $2.5 \mathrm{~mm}$

Ver. spacing: $2.5 \mathrm{~mm}$

Bad horizontal wires:

Bad vertical wires:

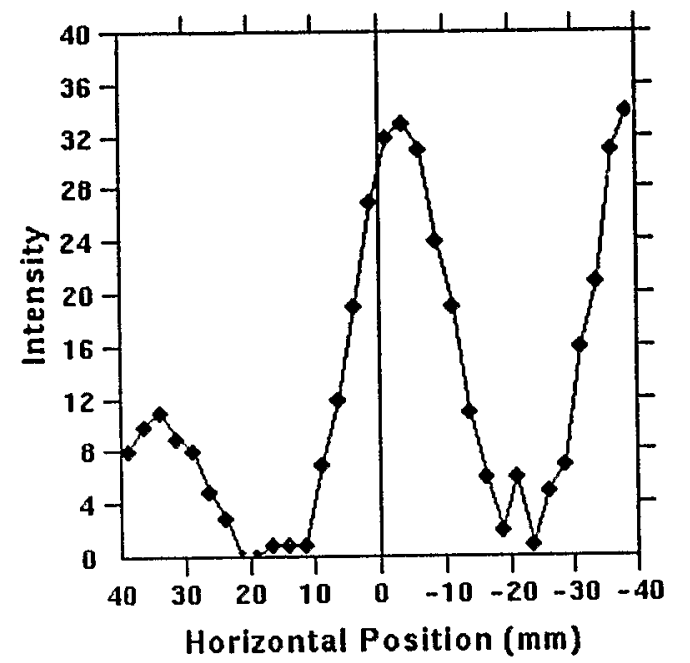

\section{HARP ABI.MW060}

Mon Apr 27 14:03:29 1992

Horizontal mean: $23.32 \mathrm{~mm}$

Vertical mean: $0.94 \mathrm{~mm}$

Horizontal FWHM: $60.83 \mathrm{~mm}$

Vertical FWHM: $11.26 \mathrm{~mm}$
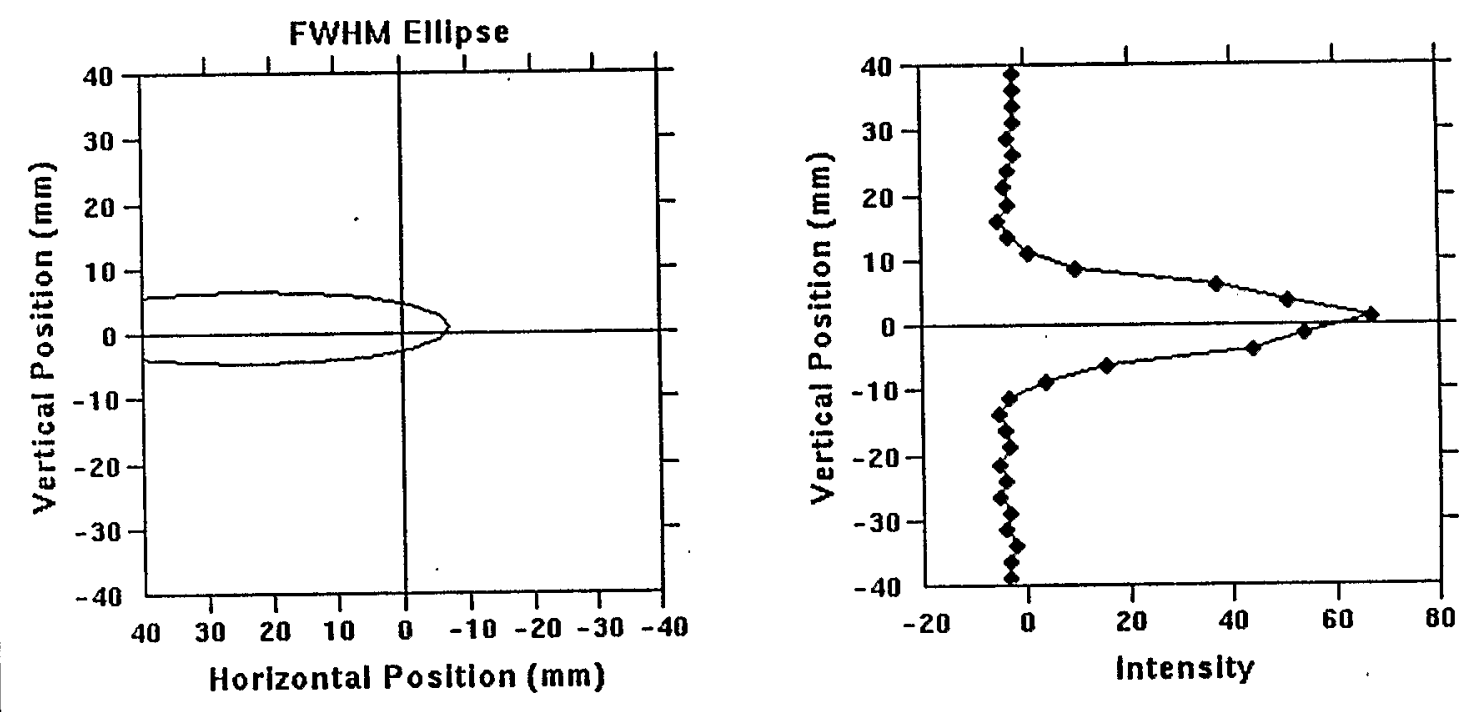
Copper Foil Thickness: $0.025 \mathrm{~mm}$

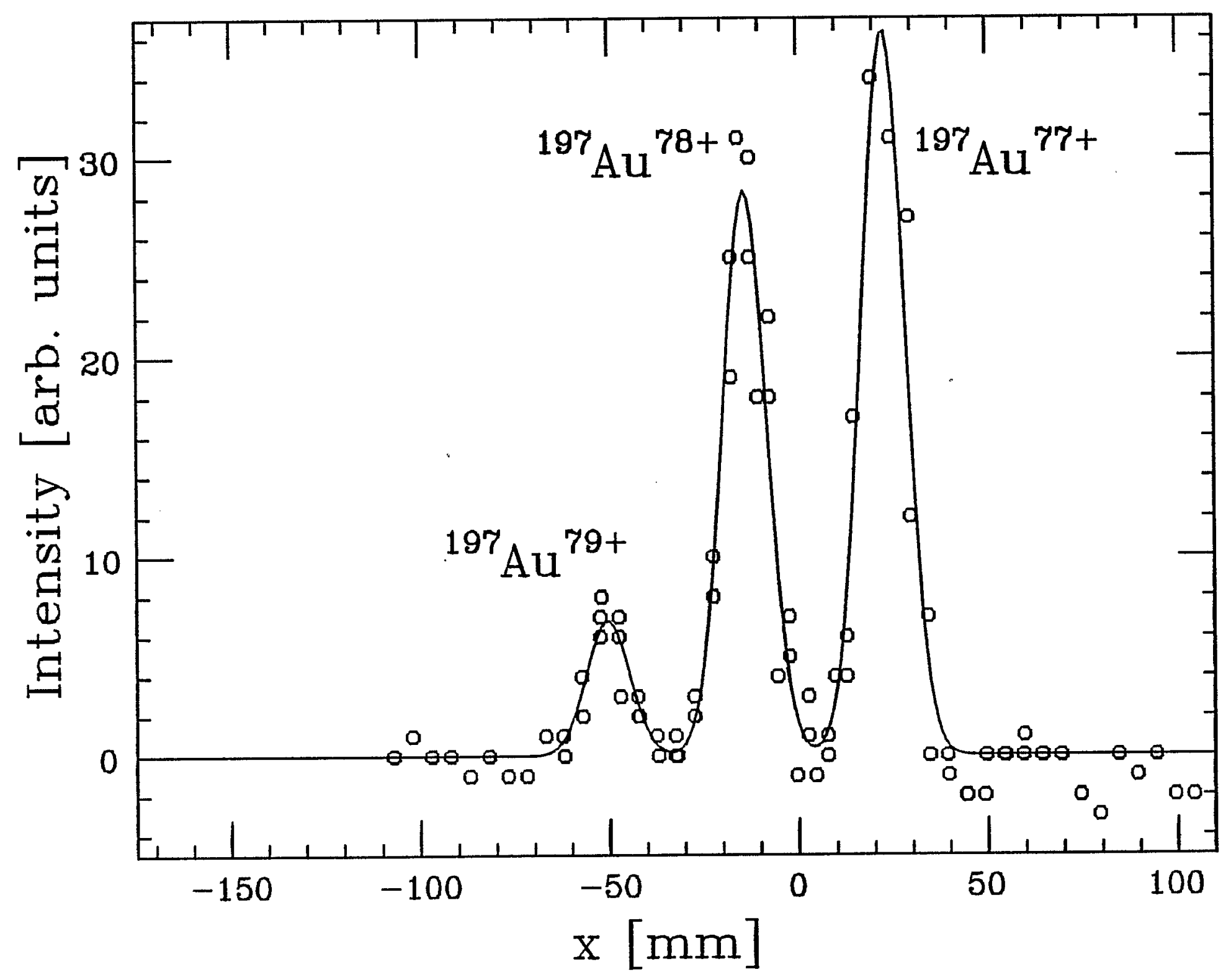


Copper Foil Thickness: $0.038 \mathrm{~mm}$

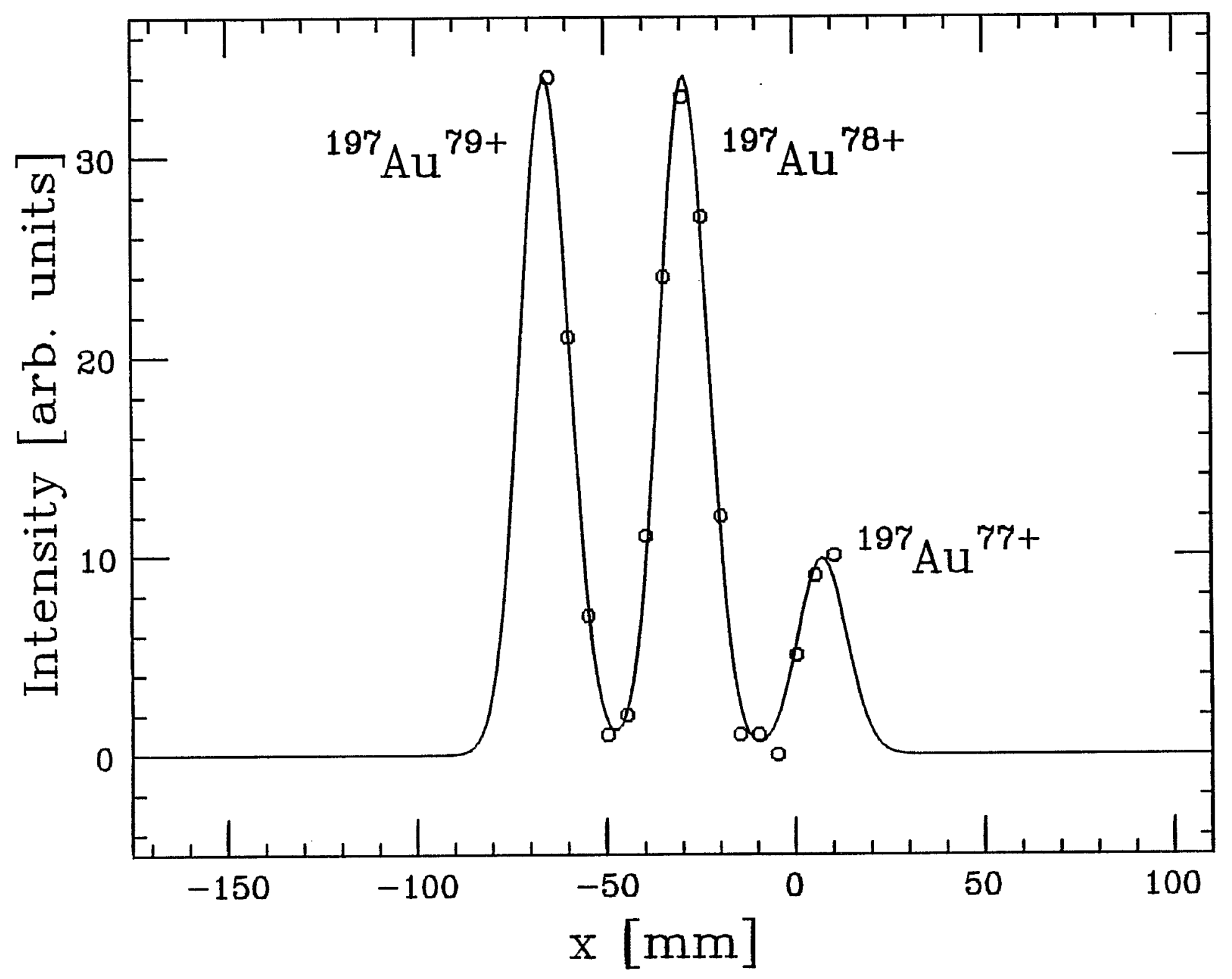


Copper Foil Thickness: $0.050 \mathrm{~mm}$

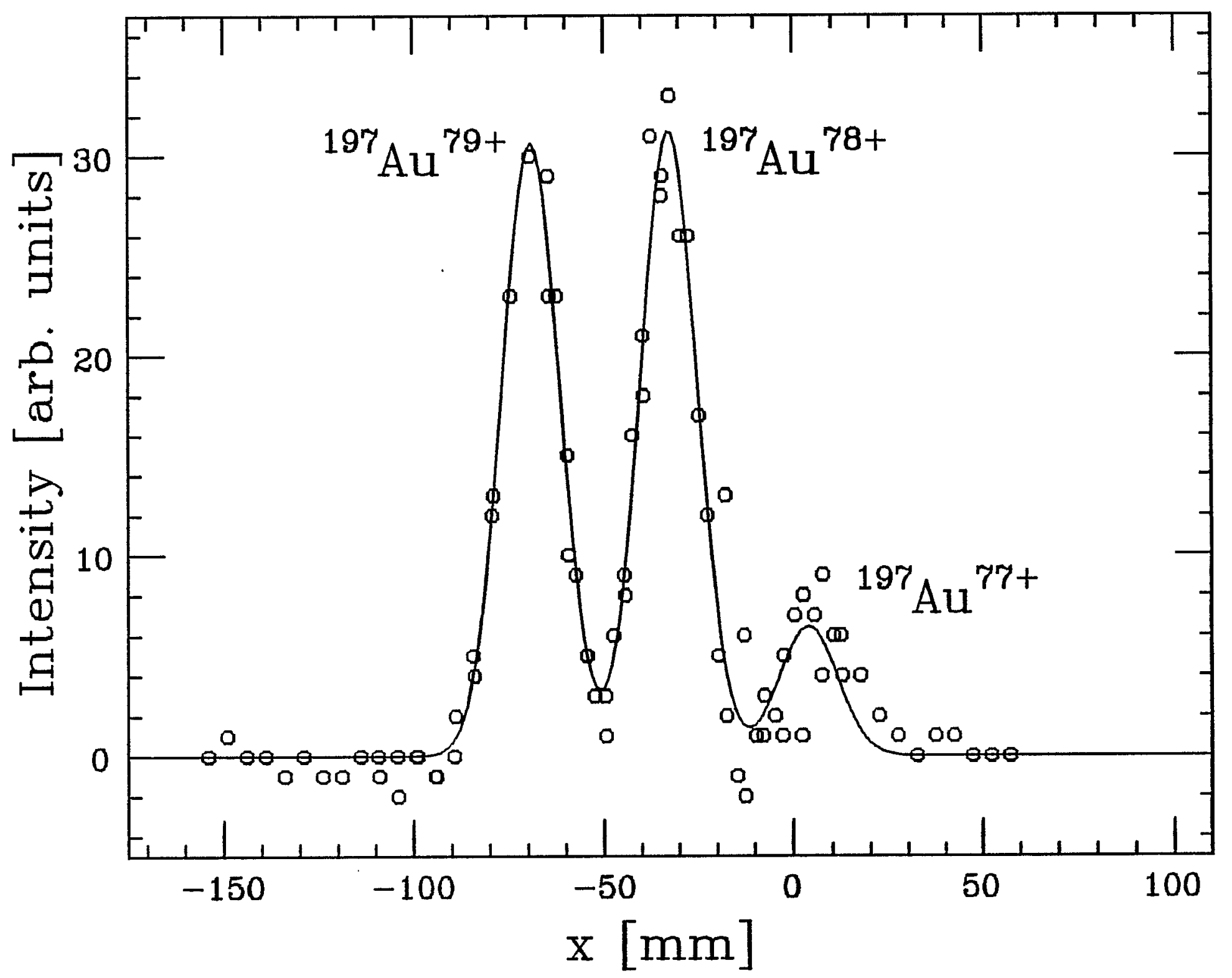


Copper Foil Thickness: $0.100 \mathrm{~mm}$

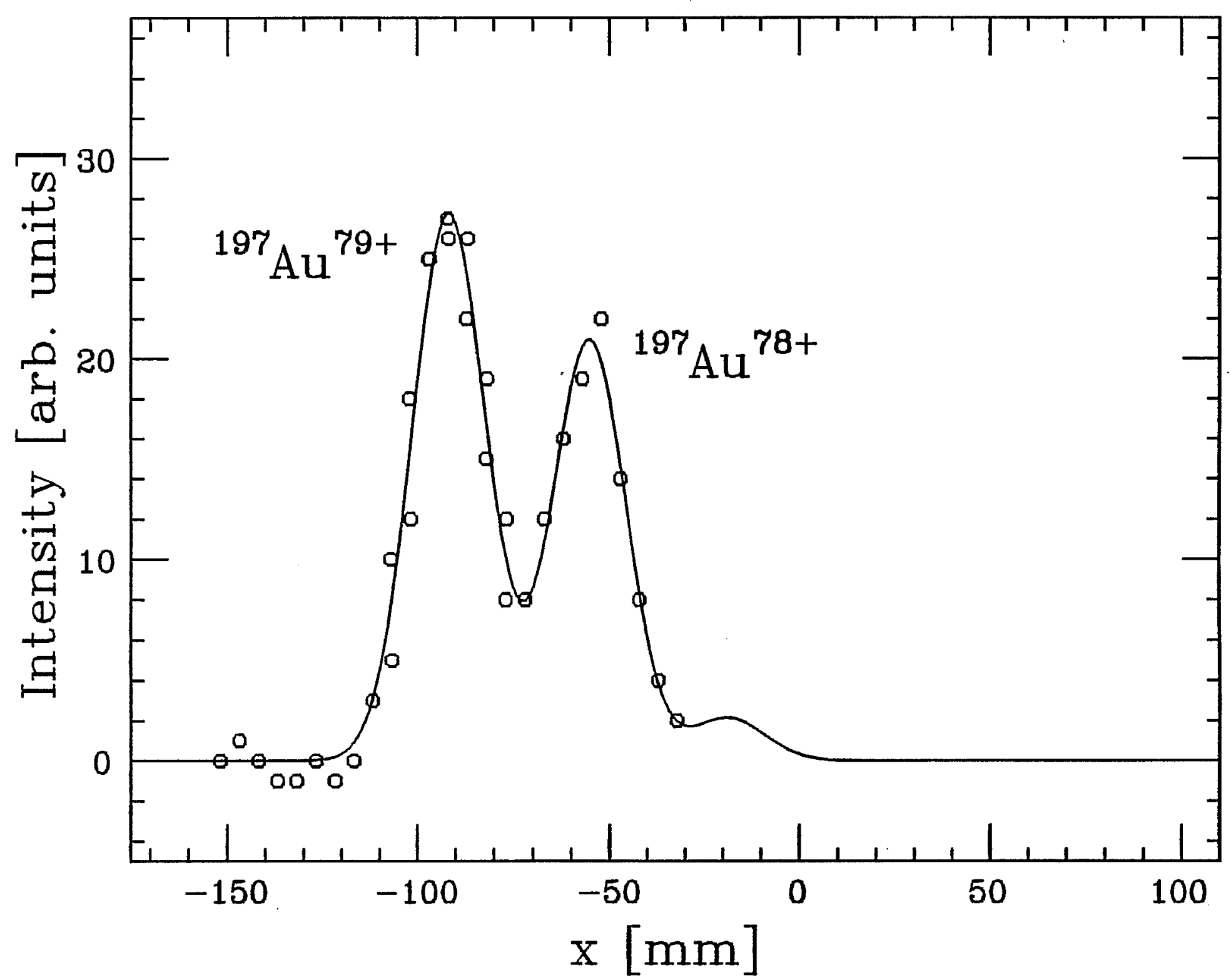




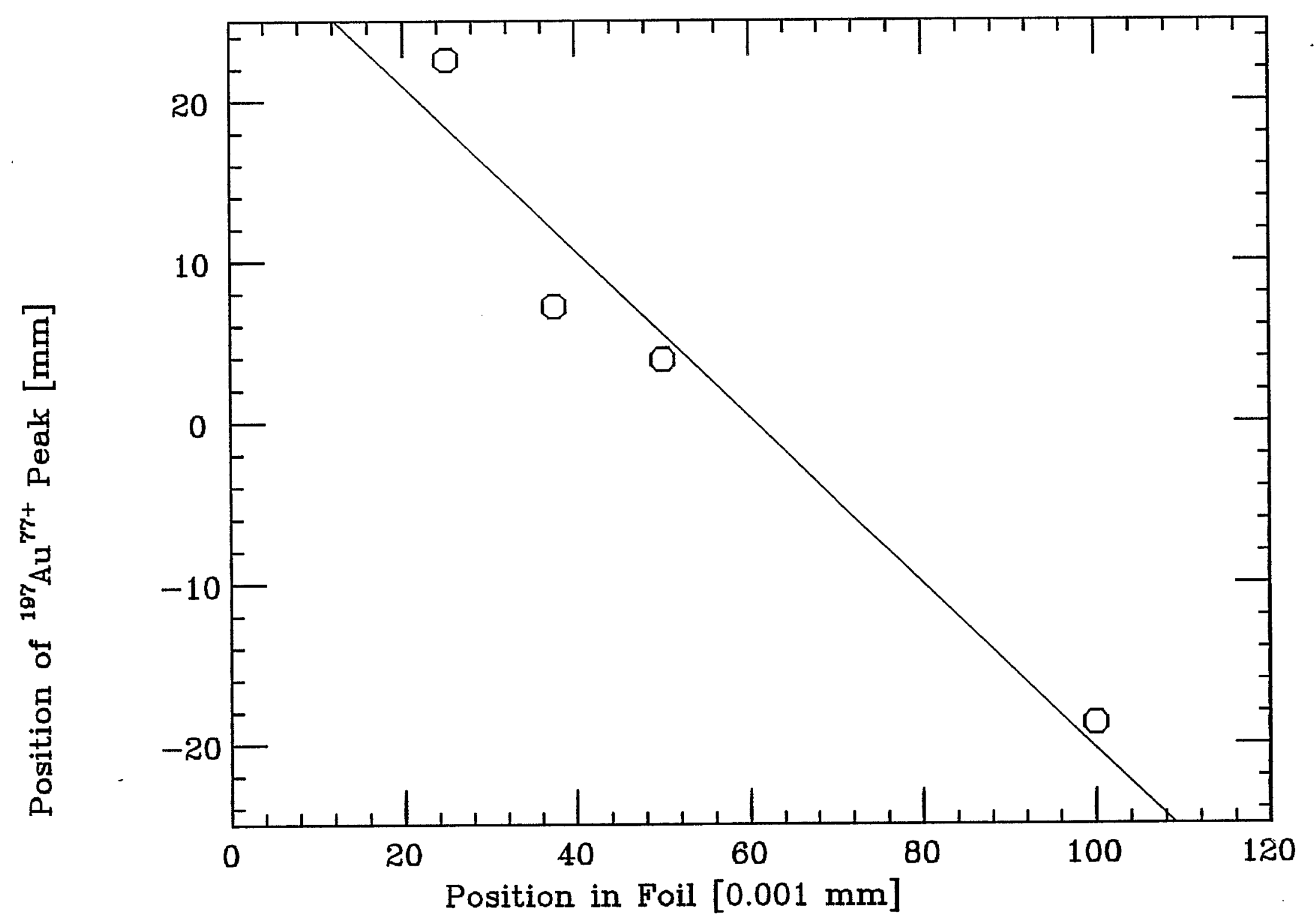




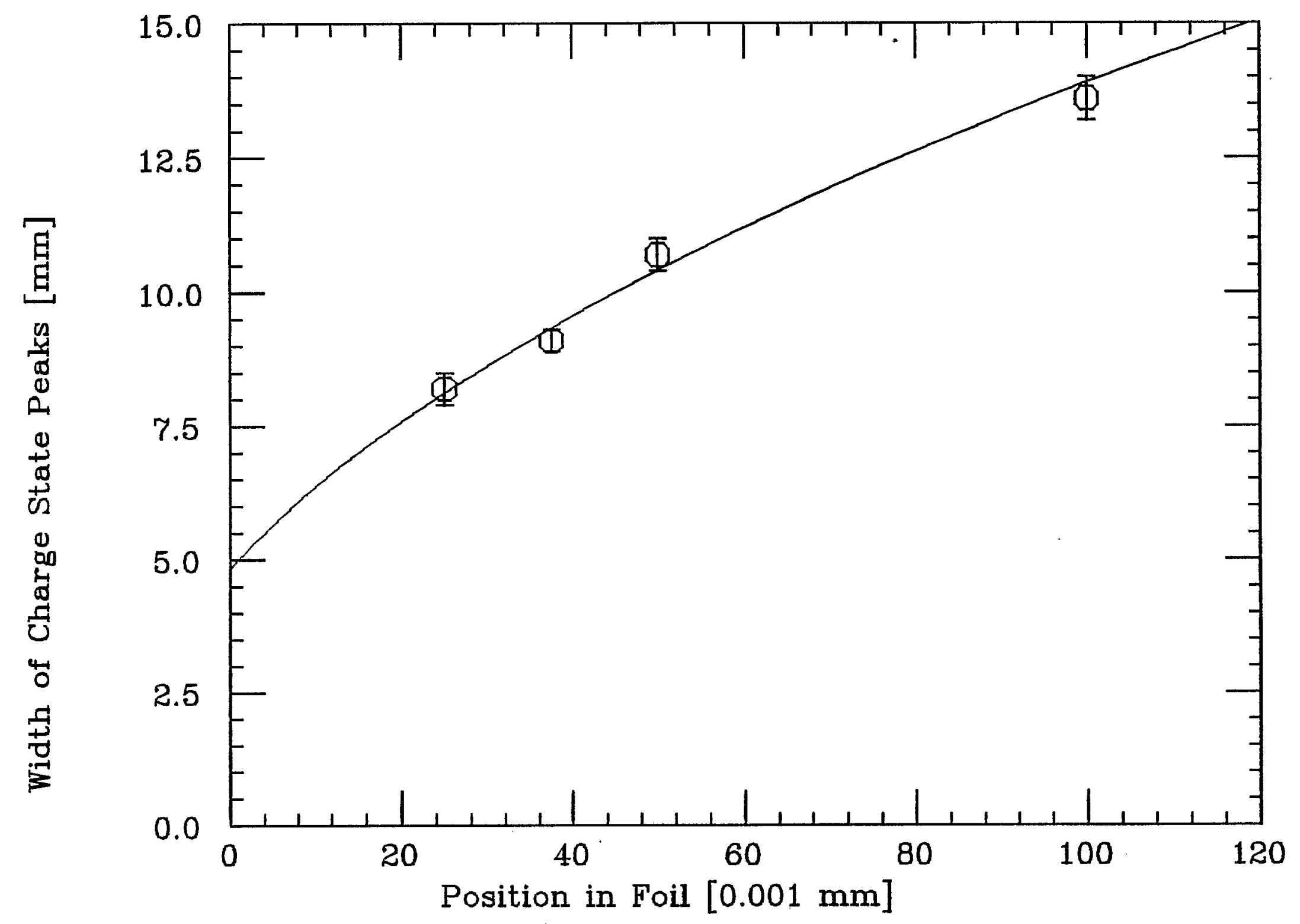




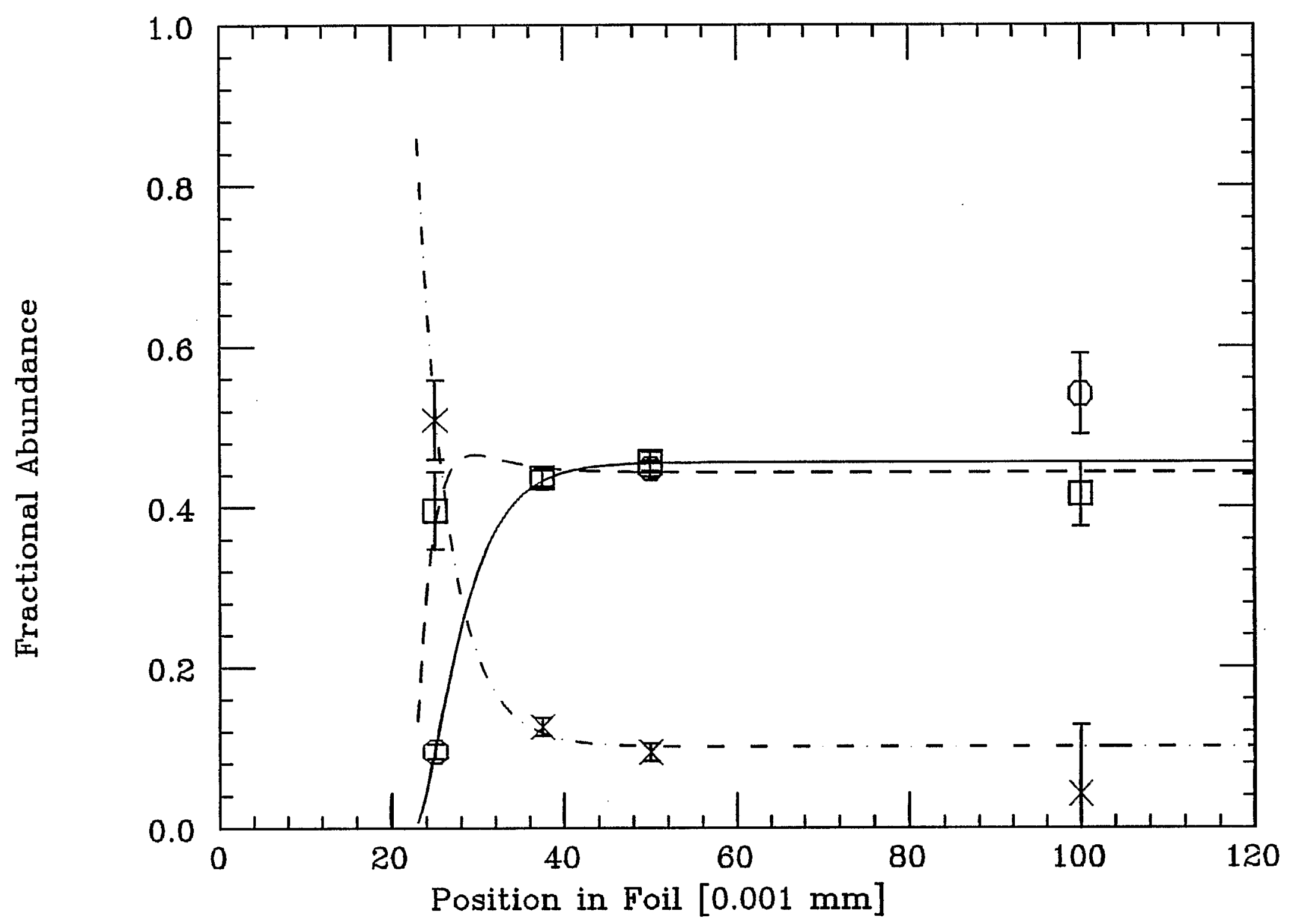

